\title{
Model of Cubic Cell for Description of Some Phase Transitions in Crystals
}

\author{
Vladimir Kh. Kozlovskiy \\ Scientific Society Berlin, WIGB, Berlin, Germany \\ Email: Kozlovskiy.V@t-online.de
}

How to cite this paper: Kozlovskiy, V.Kh. (2018) Model of Cubic Cell for Description of Some Phase Transitions in Crystals. World Journal of Condensed Matter Physics, 8, 162-170.

https://doi.org/10.4236/wjcmp.2018.84011

Received: August 1, 2018

Accepted: October 7, 2018

Published: October 10, 2018

Copyright $\odot 2018$ by author and Scientific Research Publishing Inc. This work is licensed under the Creative Commons Attribution International License (CC BY 4.0).

http://creativecommons.org/licenses/by/4.0/

\section{(c) (i) Open Access}

\begin{abstract}
The purpose of the research is to develop a dynamical theory of phase transitions in crystalline structures, when except for temperature, the pressure is acting. So, the phase diagram temperature-pressure (dimensions) must be constructed. In general case, it is a complicated question, which can be solved for simple models of crystal, as three atomic models, introduced in the work of Frenkel [1]. In this model, three identical atoms are placed on the straight line and interact with the forces, which can be described by the expression, given in the article of Lennard-Jones [2]. Such simple model may have success, when the crystalline structure is simple, which consists of one type of atoms, for example: carbon. The model was generalized to cubic cell model with a moving atom in the inner part of the cell. The rigorous calculation of phase diagram for transition graphite-diamond shows some similarity with results of numerous experimental investigations (which are not discussed here). So, the way of phase diagram calculation may attract attention.
\end{abstract}

\section{Keywords}

Cubic Cell, Phase Transitions, Influence of Temperature, Influence of Pressure

\section{Introduction}

As was mentioned in the work of Max Born, the linear or harmonic theory of crystalline lattice is insufficient to describe some properties of solid corps: structural transitions, flow, mechanical strength [3]. In order to develop the theory of these properties, introduction of anharmonic forces is necessary. The virial theorem, proved by Clausius, was used for investigation of transition in solid corps. But this theorem is insufficient for description of solid corps and behavior of atoms and must be generalized to receive more equations determining the 
atomic positions and parameters of atoms moving. This theorem was also proved in the work [4], where authors used mechanical variation principle, introducing one variation number. Introducing more variation numbers and minimizing mechanical action respective all variation numbers, gives possibility to receive a system of equations ("theorem"), which describe the behavior of atoms with sufficient accuracy. This procedure of calculation is presented in article [5]. For considering the action of high pressure (or interatomic distance) on phase transitions, Frenkel elaborated a three-atomic model, built from three identical atoms, placed on a straight line. The forces, connecting the atoms, may be considered as described by expression given in the work of Lennard-Jones. The model of Frenkel may be generalized and transformed into a cubic model, where the summits occupy immovable atoms and the atom in the inner part of the cube can occupy the centre of cube or the centre of the facet. In the first case, the cubic structure is represented, and in the second, it is flaky. At high enough temperature, an amorphous structure can arise, but for determining the place of this structure on the diagram, additions in expression of energy must be introduced. The calculated diagram can be compared with results, gained in numerous experimental investigations (which are not considered) of the transition among graphite, diamond and liquid phase of carbon. Some similarity between calculated phase diagram and constructed on the basis of experimental results can be marked, so theoretical model can be useful in research about this subject. The model of Frenkel was used in some works for examination of the phase transitions [6] [7] [8]. The present article is established on these works and is the most perfect.

\section{The Cubic Cell Model}

In Figure 1 a cubic cell model with immobile atoms in the summits and mobile atom in the inner part of the cell is shown. The origin is placed in the centre of the cell, and the coordinate axis $x, y, z$ are directed normal to the facets. The potential energy of moving atom in the inner part of the cell is represented by means of fourth power polynom of coordinates

$$
U(x, y, z)=U_{0}+\frac{c}{2}\left(x^{2}+y^{2}+z^{2}\right)+\frac{b}{4}\left(x^{4}+y^{4}+z^{4}\right)
$$

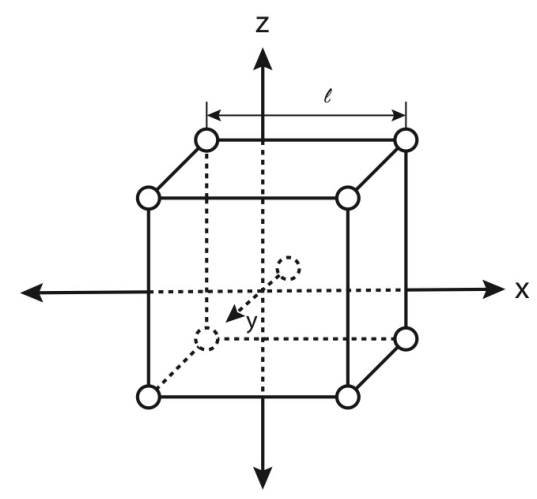

Figure 1. The cubic cell model of structural transitions. 
with the cell edge length $l$. The form of potential energy is reproduced in neighboring cells, so in two points on the $x$-axis, symmetrical placed relative to the facet, the energy has the same value, so according to Roll theorem the derivative of the energy on the facet must be zero

$$
\left(\frac{\partial U}{\partial x}\right)_{x=\frac{l}{2}}=\frac{l}{2}\left(c+\frac{b l^{2}}{4}\right)=0
$$

So

$$
b=-\frac{4 c}{l^{2}}
$$

Now we mark the values of potential energies in the centre of the cell $U_{C}$ and in the centre of facet $U_{F}$. They have values

$$
\begin{gathered}
U_{C}=U_{0} \\
U_{F}=U_{0}+\frac{l^{2}}{8}\left(c+\frac{b l^{2}}{8}\right)
\end{gathered}
$$

So it will be gained

$$
\begin{gathered}
c=\frac{16}{l^{2}}\left(U_{F}-U_{C}\right) \\
b=-\frac{64}{l^{4}}\left(U_{F}-U_{C}\right)
\end{gathered}
$$

The dependence of the coefficients $c, b$ from the cell dimensions is determined from the dependence of the Lennard-Jons potential from the distance between atoms. Taking into account only the nearest neighbors in the cell, it can be written

$$
\begin{gathered}
U_{C}=8\left[-\frac{A}{\left(\frac{l \sqrt{3}}{2}\right)^{m}}+\frac{B}{\left(\frac{l \sqrt{3}}{2}\right)^{n}}\right] \\
U_{F}=4\left[-\frac{A}{\left(\frac{l \sqrt{2}}{2}\right)^{m}}+\frac{B}{\left(\frac{l \sqrt{2}}{2}\right)^{n}}\right]
\end{gathered}
$$

where, as it will be accepted, $n=2 \mathrm{~m}$. From theses expressions it can be found

$$
\frac{1}{4}\left(U_{F}-U_{C}\right)=-A \frac{2^{\frac{m}{2}}}{l^{m}}\left[1-2\left(\frac{2}{3}\right)^{\frac{m}{2}}\right]+B \frac{2^{\frac{n}{2}}}{l^{n}}\left[1-2\left(\frac{2}{3}\right)^{\frac{n}{2}}\right]
$$

Because expressions in quadratic brackets are positive and $n$ is greater, than $m$, it follows a change of sign from positive values to negative with increasing length of the cell side. Consequently, it exist some value of length $l^{*}$, where elastic and 
anharmonic coefficients turns to zero, and the movement of the atom occurs unhampered (this question will be considered later). It can be put approximately

$$
c(l)=\left(\frac{\mathrm{d} c}{\mathrm{~d} l}\right)^{*}\left(l-l^{*}\right)+\cdots
$$

where the derivative here is negative. Further we consider the movement only along $x$-axis, because movements along all axes are identical. Taking into account (1) and (3) we write the expression of atom energy in the form

$$
U=U_{0}+\frac{c}{2} x^{2}-\frac{c}{l^{2}} x^{4}
$$

If the thermal movement is absent, the coordinate is not changed with the time and determined through the external influence. The condition of equilibrium

$$
\frac{\partial U}{\partial x}=c x-\frac{4 c}{l^{2}} x^{3}=0
$$

leads to the values of coordinate

$$
x=0 \text { and } x^{2}=\frac{l^{2}}{4}
$$

and these values correspond to the position in the centre of cell (cubic structure) and centre of facet (layered structure). The conditions of stability

$$
\frac{\partial^{2} U}{\partial x^{2}}=c-\frac{12 c}{l^{2}} x^{2}>0
$$

for the position in the centre of the cell leads to inequality $c>0$, and for the position in the centre of facet to the inequality $c<0$. We consider now the question in general case, when the change in structure occurs through the action of thermal motion and mechanical action. Then in expression (1) the coordinate as a function of time $t$ is represented in the form

$$
x=s+u \theta(t)
$$

where vibrations are described by means of simple function of time.

After the elevation of the coordinate in proper power and taking the mean time value the free energy is transformed to the form

$$
F=U_{0}+\frac{c}{2}\left(s^{2}+u^{2}\right)+\frac{b}{4}\left(s^{4}+6 s^{2} u^{2}+\overline{\theta^{4}} u^{4}\right)-k T \ln u, \quad \overline{\theta^{4}}=3
$$

The general equations of state are

$$
\begin{gathered}
\frac{\partial F}{\partial s}=c s+b s^{3}+3 b s u^{2}=0 \\
\frac{\partial F}{\partial u}=c u+3 b s^{2} u+3 b u^{3}-\frac{k T}{u}=0
\end{gathered}
$$

The general conditions of stability

$$
\text { a) } \frac{\partial^{2} F}{\partial s^{2}}=c+3 b s^{2}+3 b u^{2}>0
$$




$$
\begin{gathered}
\text { क) } \frac{\partial^{2} F}{\partial u^{2}}=c+3 b s^{2}+9 b u^{2}+\frac{k T}{u^{2}}>0 \\
\gamma) \frac{\partial^{2} F}{\partial s^{2}} \frac{\partial^{2} F}{\partial u^{2}}-\left(\frac{\partial^{2} F}{\partial s \partial u}\right)^{2}=\left(c+3 b s^{2}+3 b u^{2}\right)\left(c+3 b s^{2}+9 b u^{2}+\frac{k T}{u^{2}}\right)-(6 b s u)^{2}>0
\end{gathered}
$$

From equation (18), equation (19) follows two expressions, describing the states conditionally called symmetrical $(s=0)$ and nonsymmetrical $(s \neq 0)$. Taking the amplitude as independent variable, one can write the expressions of displacement and temperature

$$
\begin{gathered}
s=0, \quad k T=c u^{2}+3 b u^{4} \\
b s^{2}=-c-3 b u^{2}, \quad k T=-2 c u^{2}-6 b u^{4}
\end{gathered}
$$

In Figure 2 and Figure 3 are shown the dependencies of temperatures from amplitude for both structures.

The coordinates of maximum in Figure 2 are the following $(c>0, b<0)$

$$
u_{M}^{2}=-\frac{c}{6 b}=\frac{l^{2}}{24} ; \quad k T_{M}=-\frac{c^{2}}{12 b}=\frac{c l^{2}}{48}
$$

The conditions of stability (20) and (21) are formulated as

$$
u^{2}<-\frac{c}{3 b} ; \quad u^{2}<-\frac{c}{6 b}
$$

From these inequalities essential is the second that marks the part of the curve to the left side of maximum as stable one and the inequality (22) is a consequence of precedents. The coordinates of maximum in Figure 3 are the following $(c<0, b>0)$

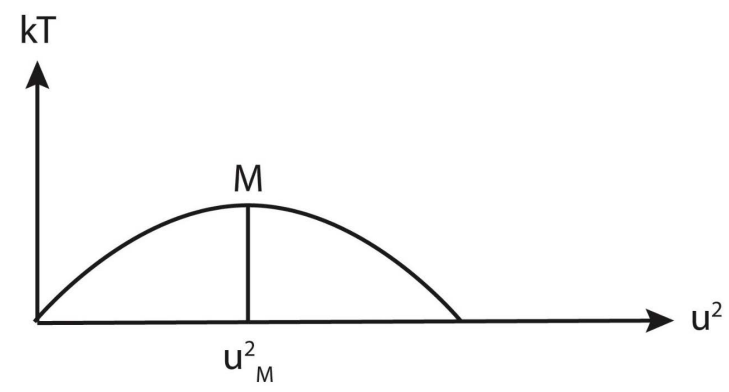

Figure 2. The dependence of temperature from amplitude for symmetrical structure.

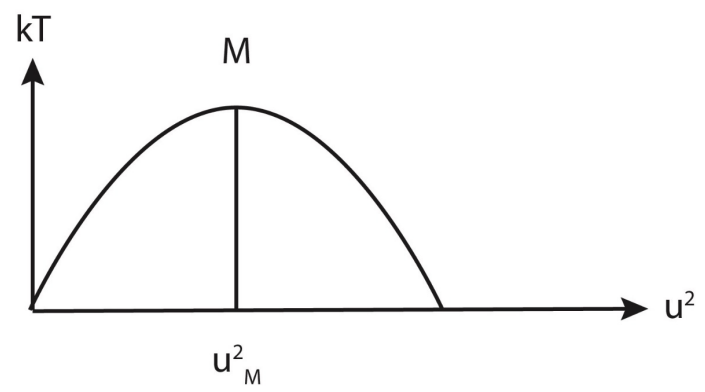

Figure 3. The dependence of temperature from amplitude for non symmetrical structure. 


$$
u_{M}^{2}=-\frac{c}{6 b}=\frac{l^{2}}{24} ; \quad k T_{M}=\frac{c^{2}}{6 b}=-\frac{c l^{2}}{24} ; \quad s_{M}^{2}=-\frac{c}{2 b}=\frac{l^{2}}{8}
$$

Excluding the displacement and reducing we receive the conditions of stability for non symmetrical structure in the form

$$
\begin{gathered}
-c-3 b u^{2}>0 \\
-2 c-3 b u^{2}>0 \\
\left(-c-3 b u^{2}\right)\left(-c-6 b u^{2}\right)>0
\end{gathered}
$$

So the inequality shown in formula (31)

$$
u^{2}<-\frac{c}{6 b}
$$

denotes the stable part of the curve. It is possible to express the temperature of maximum $T_{M}$ through cell dimension $l$, which is affected to the action of pressure. This temperature is the highest temperature, which can exist in considered structure without transition in the other state (it is possible a transition in the liquid state). In Figure 4, the graph of functions describing transitions for symmetrical and non symmetrical structures is shown (phase diagram).

According to diagram it can be concluded that when $c$ is zero the liquid state exists at zero temperature. But such result is a consequence of simplifications in choice of model, where some potential barriers are not taken into account, but can be caused by defects of the structure. Their presence may be taken into account phenomenological through introducing additional terms in the energy and instead of (12) now will be written more general expression

$$
U=U_{*}+\frac{c+\varepsilon}{2} x^{2}+\frac{b+\beta}{4} x^{4}, \varepsilon>0, \beta<0
$$

without any relation between new constants $\varepsilon, \beta$. The following calculations will be the same, and for temperature of transition may be taken the expression (25), where for symmetrical state $c>0$

$$
k T_{M}=-\frac{(c+\varepsilon)^{2}}{12(b+\beta)}=\frac{l^{2}}{48} \frac{(c+\varepsilon)^{2}}{c-\frac{\beta l^{2}}{4}}
$$

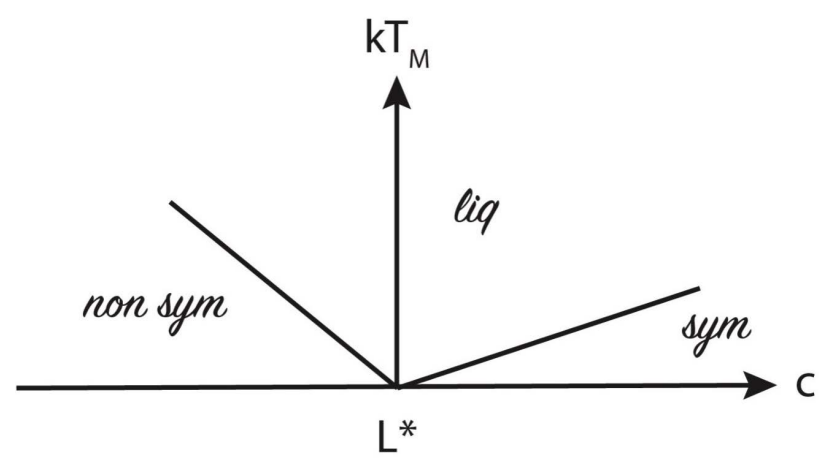

Figure 4. Phase diagram for three atomic models. 
The dependence of $k T_{M}$ from $c$ is reproduced by means of mathematical function $y(x)$ of the form

$$
y=\frac{(x+p)^{2}}{x+q}, \quad p>0, \quad q>0
$$

graph of which is shown in Figure 5. The value in the origin is non zero but $\frac{p^{2}}{q}$ and may be equal to the corresponding value for non symmetric state through the choice of new constants. For non symmetrical state $(c<0)$ according to (27), we receive (new constants may have now opposite sign)

$$
k T_{M}=\frac{(c+\varepsilon)^{2}}{6(b+\beta)}=\frac{l^{2}}{24} \frac{(-c-\varepsilon)^{2}}{-c+\frac{\beta l^{2}}{4}}
$$

Very schematic diagram with marked regions of diamond, graphite and liquid carbon existence is represented in Figure 6. This diagram is received on the base of experimental synthesis of diamond from graphite, and some resemblance with calculated one may be marked [9]-[22]. It is essential, that the line of transition diamond-graphite is not a vertical line perpendicular to the line of pressure, but is banded to the line of pressure and shows conjointly increase of temperature and pressure. Such behavior is characteristic for melting phenomenon and has its reason in increase of barrier heights for jump of an atom through the interval between the neighbors. Graphite in relation to diamond behaves like a liquid and for such behavior are reasons. The density of graphite is less, than the density of diamond, the carbon nets in graphite are far removed one from another,

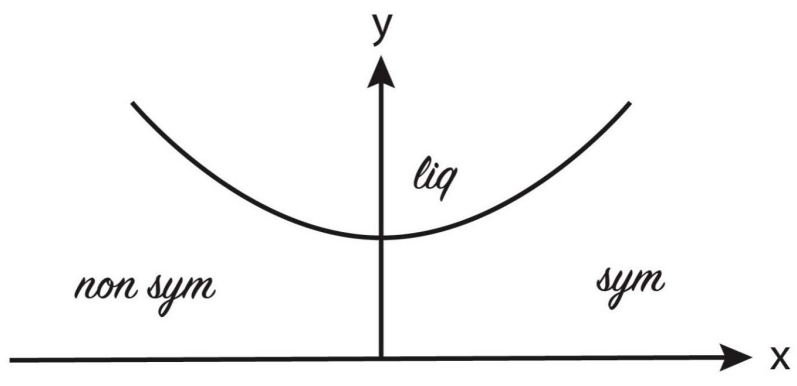

Figure 5. Mathematical graph of phase line.

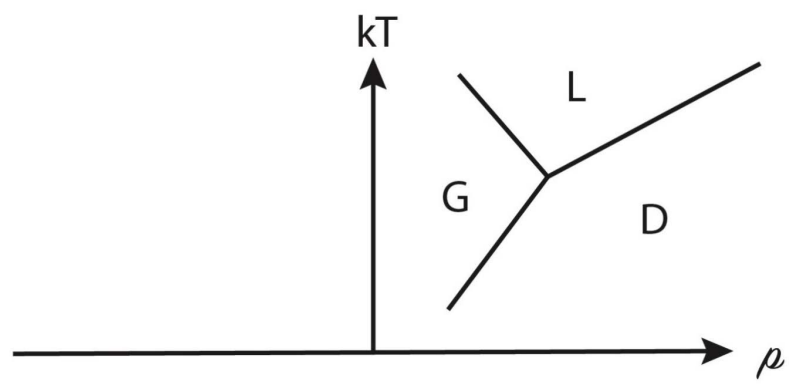

Figure 6. Phase diagram temperature-pressure for carbon. 
their mutual interaction is week, so they glide relative one another like molecules. But graphite in relation to liquid carbon behaves like a crystalline corps, which temperature of melting falls with increase of pressure. Graphite is a soft material and may be changed under the action of pressure. It exist an exploration, where graphite was exposed to $\gamma$ radiation before utilization for diamond synthesis [23]. His structure was changed and the quantity of diamond diminished. It can be marked, that in article [24] the line of equilibrium between two solid phases is shown as nearly normal to the line of pressure.

\section{Concluding Remarks}

The result of calculations on the basis of dynamical theory of phase transitions is the phase diagram temperature-pressure (or cell dimensions) for elaborated here cubic model of Frenkel. Comparison with diagrams, constructed on the basis of numerous experimental investigations of phase transition graphite-diamond shows some resemblance between calculated and experimental diagrams. So, the model of Frenkel can serve not only as illustration possibility of phase transitions, but can be used in researches. It can be supposed that a combination of such models in linear chain can give possibility to come near to such phenomenon as rupture and some other anharmonic effects.

\section{Conflicts of Interest}

The author declares no conflicts of interest regarding the publication of this paper.

\section{References}

[1] Frenkel, J. (1935) The Liquid State and the Theory of Fusion. Acta Physicochimica URSS, 3, 633-648.

[2] Jones, J.E. and Ingham, A.G. (1925) On the Calculation of Certain Crystal Potential Constants, and of a Cubic Crystal of Least Potential Energy. Proceedings of the Royal Society of London A, 107, 636-653. https://doi.org/10.1098/rspa.1925.0047

[3] Born, M. (1951) The Limit of Ideal Crystal Theory Applicability and Their Overcoming. Ceremonial Assay to the Celebration of Two Hundred Years Creation Academy of Sciences in Goettingen, Springer Publishing, Berlin.

[4] Fock, V. and Krutkow, G. (1932) Remarks to the Virial Theorem of Classical Mechanic. Physical Magazine of USSR, 1, 756-758.

[5] Kozlovskiy, V.Ch. (1991) Dynamical Equations for Time-Averaged Coordinates at Thermal Equilibrium. Soviet Physics Journal, 34, 353-359.

https://doi.org/10.1007/BF00898103

[6] Kozlovskiy, V.Ch. (1969) Phase Diagram Length-Temperature for Three Atomic Model of a Crystal. Mechanism and Kinetic of Crystallization. Science and Technic, Minsk, 154-163.

[7] Kozlovskiy, V.Ch. (1999) Dynamical Consideration of Mechanical Behavior According Three Atomic and Melting According Nine Atomic models of a Crystal. Transactions of IV International Conference "Crystals. Growth, Properties, Real Structure, Applications", 2, 466-476. 
[8] Kozlovskiy, V.Ch. (2003) Vibration-Diffusion Model of Melting. Transactions of VI International Conference "Crystals. Growth, Properties, Real Structure, Applications", 579-594.

[9] Leipunskij, O.I. (1939) About Imitation of Diamonds. Progress in Chemistry, 8, 1519-1531.

[10] Sobolew, W.S. (1960) Condition of Diamonds Layer Formation. Vol. 1, Institute of Geology and Geophysics, Siberia Department of Science Academy USSR, 7-22.

[11] Bundy, F.P., Bovenkerk, H.F., Strong, H.M. and Wentorf, R.H. (1961) Diamond-Graphite Equilibrium Line from Growth and Graphitization of Diamond. The Journal of Chemical Physics, 35, 383-391. https://doi.org/10.1063/1.1731938

[12] Bundy, F.P. (1962) Melting Point of Graphite at High Pressure. Heat of Fusion. Science, 137, 1055-1057. https://doi.org/10.1126/science.137.3535.1055

[13] Bundy, F.P. (1962) Direct Conversion of Graphite to Diamond in Static Pressure Apparatus. Science, 137, 1057-1058. https://doi.org/10.1126/science.137.3535.1057

[14] Bundy, F.P. (1963) Melting of Graphite at Very High Pressure. Journal of Chemical Physics, 38, 618-630. https://doi.org/10.1063/1.1733715

[15] Bundy, F.P. (1963) Direct Conversion of Graphite to Diamond in Static Pressure Apparatus. Journal of Chemical Physics, 38, 631-643. https://doi.org/10.1063/1.1733716

[16] Wasiliew, W.G., Kowaljskij, W.W. and Tcherskij, N.W. (1961) Problem of Diamond Origin. Yakut Publishing House, Yakutsk.

[17] Wereshtshagin, L.F. and Fateewa, N.S. (1971) To the Question about the Curve of Graphite to $90 \mathrm{kbar}$ Melting. Lettersw to the Journal of Experimental and Theoretical Physics, 13, 157-159.

[18] Tonkow, E.J. (1979) Phase Diagrams of Elements at High Pressure. 38-41.

[19] Nowikow, N.W., Fedoseew, D.W., Shulgenko, A.A. and Bogatirewa, G.P. (1987) Synthesis of Diamond. Scientific Meditations, Kiev, 10-32.

[20] Andreew, B.D. and Malik, W.R. (1985) Method of Calculation Thermodynamically Functions and Graphite-Diamond Equilibrium Curve in Wide Range of Temperatures. Influence of High Pressure on Structure and Properties Super Hard Materials. Collection of Scientific Works. Kiev, 12-19.

[21] Digonskiy, W.W. and Digonskiy, S.W. (1992) Regularities of Diamond Formation. Sankt-Peterburg. "Entrails".

[22] Sawwatimskiy, A.I. (2003) Melting of Graphite and Liquid Carbon. Successes in Physics Sciences, 173, 1372-1379.

[23] Dutow, A.G., Asarko, I.I., Koslova, E.I., Komar, W.A., Kurilowitsh, N.F., Shipilo, W.B. and Shipilo, N.W. (2006) Spontaneous Synthesis of Diamond Crystals from Graphite Irradiated by Means of $\gamma$-Quants. Crystallography, 51, 130-135.

[24] Stishow, S.M. (1968) Melting at High Pressures. Successes in Physical Sciences, 96, 467-494. 